\title{
Augmented Reality Painting and Collage: Evaluating Tangible Interaction in a Field Study
}

\author{
Giulio Jacucci ${ }^{1}$, Antti Oulasvirta ${ }^{1}$, Antti Salovaara ${ }^{1}$, Thomas Psik ${ }^{2}$, and Ina Wagner ${ }^{2}$ \\ ${ }^{1}$ Helsinki Institute for Information Technology, \\ P.O. Box 9800, FIN-02015 TKK, Finland \\ \{firstname.surname\} @hit.fi \\ ${ }^{2}$ Vienna University of Technology, \\ Argentinierstrasse 8, A-1040 Vienna, Austria \\ iwagner@pop.tuwien.at, thomas.psik@media.tuwien.at
}

\begin{abstract}
Tangible computing applications are rarely evaluated with field studies in real settings, which can contribute as formative studies to understand the challenges and benefits of tangible interfaces in real world practices. We present an AR environment for painting, with a physical brush, digital textures on physical models and creating dynamic stages for the model with spatial collages providing different backgrounds. We report on an evaluation of this AR environment in an architecture school, where 8 groups of students used it as a practical assignment. The evaluation demonstrated the benefits of specific features of the environment and of its tangible interfaces: immersiveness, public availability, supporting collaboration, flexibility, dynamicism and resulting rapidity in creating mixed media representations. Several challenges surfaced from the evaluation especially in connection to the distribution of the interface. The physical, spatial, and computational separation of interface components raised issues on accountability and ergonomics. We link our observations to design guidelines.
\end{abstract}

\section{Introduction}

Research on developing augmented environments has been rarely based on naturalistic field trials. Various reasons have been indicated, for example, the difficulty of producing prototypes reliable enough to be introduced in real settings, as they often include the widest range of technology that has to work together: software, middleware, hardware, and physical interface (cf. [1]).

This is also part of a general trend, as Abowd and co-authors indicate that little research is "published from an evaluation or end-user perspective in the ubicomp community" [2, p. 56]. Naturalistic studies in real settings are important not only as summative empirical studies, but also as formative studies that can inform the development of applications and interfaces, especially in "pervasive computing in which technologies' potential purposes are not clear". Moreover, even if the purpose is clear, the fits or benefits of a specific application or interface can be only validated through a naturalistic study and specific challenges might only emerge in real use. Following this direction, we report here on a naturalistic evaluation of and environment for AR painting and collage. We use the concepts of "naturalistic or situated evaluation" and 
"real setting" to mean that the technology was used by participants in their own physical environment, to carry out their own projects and goals.

In this AR environment, users can position a physical model on a table, for example on top of a projected plan, and use an application, the Texture Brush, for "painting' the model using various digital textures. The Texture Brush uses a real brush equipped with a button, which is tracked by an infrared camera. The system projects the virtual 'paint' only where the brush passes by and the button is pressed. In addition, the user can configure an immersive and dynamic stage for the model with three large projections in the background. A simple barcode scanner interface makes it possible to load digital media as texture to be painted or as background. Moreover, painted textures and the background stage can be saved as configurations on a simple barcode, making it possible to load and save sessions with a simple scan of a barcode. After the related work, we present in Section 2 the environment and its components. In Section 3, we report the main findings from the field study, which included 8 groups of architecture students alternatively working in the environment over a period of two weeks. In Section 4, we summarise the lessons learned from the evaluation in terms of the benefits of this tangible interface and of the challenges and problems that emerged during the study.

\subsection{Related Work}

The related work can be described as being part of these different categories: application demonstrations, comparisons of 2D versus tangible, observational studies, heuristic evaluation and system guidelines.

Application Demonstrations. Physical models have been used as canvases for digital images previously, for example in [3]. Their system, designed with architects and designers in mind, projects imagery onto scale models and contains a real-time location tracking. This allows users to move objects around the table, while the projection is adjusted to follow the movements. Users manipulate the projected images by using a graphics program on an adjacent computer [3]. Another system has a stylus that enables the user to work with the model directly [4]. This model also lets the user move the objects around and hold them in their hands. There is a palette projected onto a table where the user may choose the way he/she manipulates the projection with the stylus. Limitations of the approach, according to the authors, include occlusion and shadows in the model, and the question of catering for objects whose shape may change. Another work reports on a system for learning the principles of pneumatics [5]. In this case, two cameras are positioned over a table, and barcode image recognition is applied to identify the objects on the table, thus enabling the showing of simulations of flow in pneumatic networks as an image overlay. Another similar system visualizes how a laser travels through prisms positioned on the table [6]. The I/O Brush [7] takes an alternative approach. The brush includes a video camera that lets users scan patterns from their environment and paint them onto a digital canvas. The system was developed for children to explore drawing and their surrounding environment.

Comparisons of $2 D$ vs TUIs. The intuitiveness of basic user interface manipulation actions [9] and cognitive support for spatial layout tasks [10]. In relation to interaction 
and manipulation, the results from controlled experiments suggest the benefits of 3D over 2D interaction in spatial reasoning, [10] and physical interfaces over virtual models $[10,9]$. An early study on graspable user interfaces in a multi-target tracking task also suggested specialization of each interface tool so as to serve only one function in a system, as opposed to being a general-purpose controlling tool [11].

Heuristic Evaluations. User requirement and evaluation oriented studies in the field of ambient media have covered the usability and effectiveness of ambient displays [8].

Observational Studies. Open-ended observation studies include an experiment on problem solving in instructed engineering tasks [12], where it was found that physical objects provide situational support for thinking. Naturalistic studies on the use of tangible user interfaces remain very rare and are increasingly needed in order to move interface research beyond what is merely possible to implement towards tangible interfaces for real world use.

These works move from different research approaches and do not provide grounding or investigations into the concept or requirements behind the application from a user point of view, nor do they advance our knowledge in terms of what the challenges and agendas for tangible interfaces are (an exception is [20], which will be examined in the discussion). In addition to addressing these issues our work contributes, to the discussion on how previous system guidelines on table-top displays [20] can be relevant in TUI environments and how they need to be extended and modified.

\section{An Environment for AR Painting and Collage}

\subsection{Components of the Environment}

The environment for AR painting that we have developed, supports users in mixing digital media and physical models in an immersive, multi projection set-up.

The main components of the environment are:

- The hypermedia database. Users can upload to the database, pictures and videos, which are used to work with their models. When the media files are stored in the database, print-outs are created with thumbnails and barcodes to retrieve the pictures and videos during use (Figure 1a).

- The Texture Brush. This is the application that enables the user to paint digital texture on models using a physical brush (Figure 1b). The hardware includes a data projector, an infrared tracking system, and a physical brush augmented with a wireless button and a retro-reflecting surface.

- Large projection screens. Three projectors are used to play media on large projector screens behind the models (Figure 1b).

- The barcode configuration poster. A barcode Reader is used to perform basic configuration commands in the environment. 

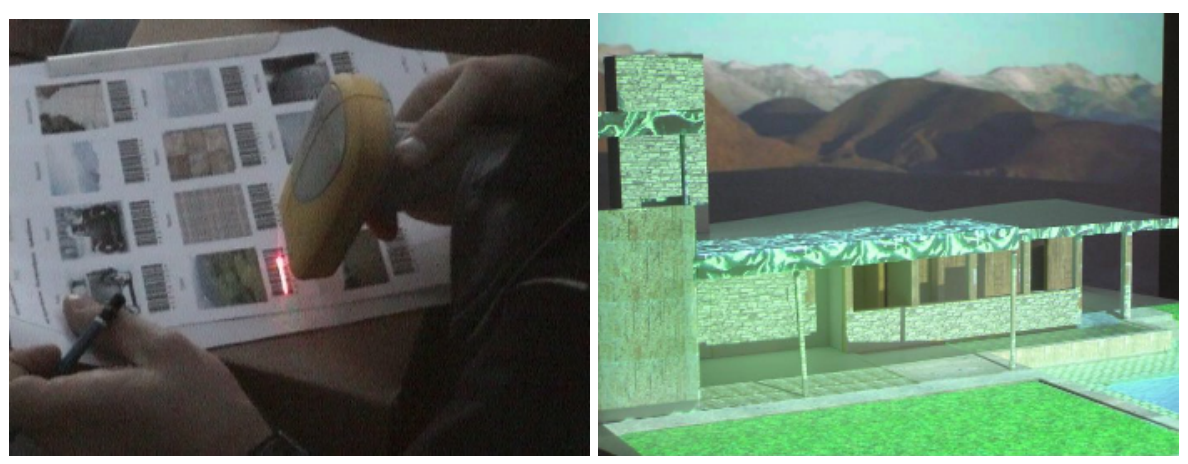

Fig. 1. From left: a) A print out with thumbnails of media files and barcodes. b) an example of a painted model with a large projection creating a background

\subsection{The Texture Brush}

With the Texture Brush design, users are able to 'paint' various computer generated visual overlays as textures onto physical 3D models in real time. Different textures are associated with different texture-samples and the actual painting is done by moving the physical "paint-brush" and tracking its position on the physical model. One of the first prototypes of the Texture Brush used a video camera for tracking the physical brush. The lightning conditions were often critical and interfered with the architectural model, so we had to use a white spot, generated by software, to light the retroreflecting surface of the brush. This spot interfered with the reception of the model. The system was also too slow with this kind of tracking technique. We decided to switch to a professional solution based on infrared technology. With this tracking device, we get faster tracking $(60 \mathrm{~Hz})$, the tracking module needs much less $\mathrm{CPU}$ power, it is easier to install for the user and we get tracking results with higher precision. Users can configure the Texture Brush in many ways. They can manipulate the brush size and shape by selecting these attributes from a menu bar, located at the bottom of the projection area, which is constantly displayed. Working tools like "polygon fill" that are familiar from applications like Adobe Photoshop@, have been integrated into the Texture Brush application. This allows the students to work with the Texture Brush in much the same way they are used to working with applications they know.

They can choose from a number of textures to use, including animated textures (videos), and the projection of the textures can be moved, scaled and rotated. The whole interaction is done using the brush as the only input device. Barcodes can be used to load the textures into the system at run-time. Any image or video that is stored in the Hypermedia database, can be used as a texture to paint the objects with.

A menu bar displays the textures along with four main menu elements. These elements are displayed as a status bar, located at the bottom of the projection area (it is displayed on the vertical border of the table). The menu elements are palette, brushtype/size, and transform (Figure 2): 
- From the palette, the user is able to select from ten different layers of textures.

- From brush-type/size, the user is able to specify brush type, brush size and shape of the brush. Flat brushes, round brushes or square brushes will be available. There is a function to create a polygon and fill it with a particular texture. Once an area/polygon is specified it will be filled at once. This helps to paint large areas in one step.

- From the "transform" menu, the user finds functions for transforming the displayed textures. The menu element "scale" allows the user to enlarge or downsize the texture by moving the physical brush at various distances from the model. "Rotate" can turn the texture clockwise or counter clockwise by rotating the physical brush.
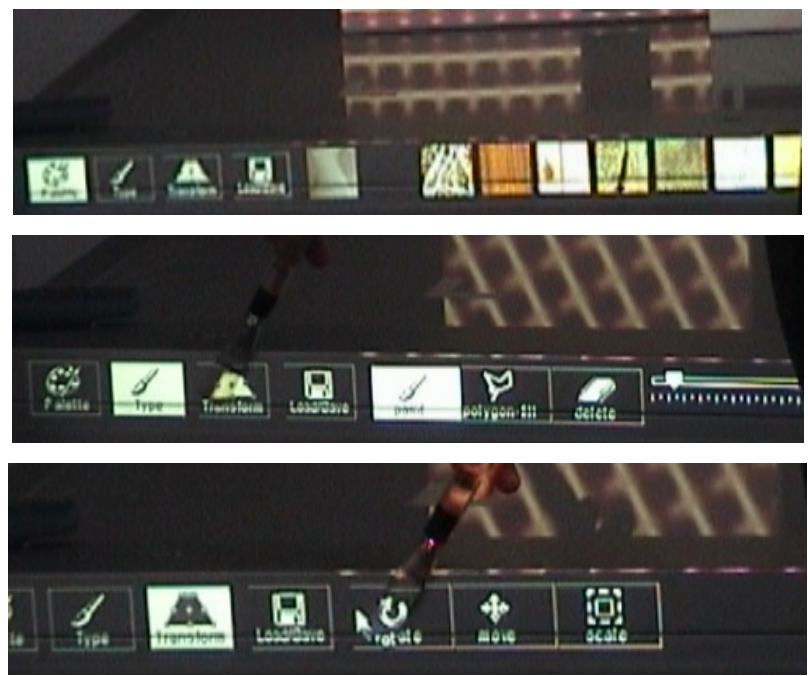

Fig. 2. From above: a) the menu item palette displays all the textures to choose from. b) the menu item "type" with brush, polygon fill, delete, and a bar to adjust the size. C) "transform" with the possibility to rotate, move, and scale.

\subsection{The 'Configuration Poster'}

Configuration of the space with such a variety of projection possibilities is not trivial. Students have to configure the system so that specific images are projected onto a specific projection screen, or used as a texture in the texture brush. We have designed a simple physical handle to configure the space a configuration poster with a variety of barcodes corresponding to basic configuration and commands:

- Specify an output (texture brush or one of the back projections)

- Saving and loading sessions

Users can specify the receiver of a texture (a picture or video) or any other input. A poster displaying the possible connections between inputs and outputs using barcodes can be used to configure the system. There is a barcode for each command, the bar- 
code reader input can be used to load the media file associated with a specific barcode as a texture with the Texture Brush display.

Additional barcodes have been added to specify printers and background projections on the cave corner as other output components. Other barcodes on the poster serve to save configurations of painted textures on empty barcodes. These connections between input and output persist as long as they are not reconfigured by the students. Configuration and re-configuration can be performed at any time, dynamically changing the set-up of the workspace, using only the configuration poster, barcode reader, and barcodes that correspond to the images stored in the database.

\section{Evaluating Tangible Interaction in a Field Study}

\subsection{Method and Analysis}

The approach that guided this field study and its analysis was work-oriented design, this includes combining: "in situ interviewing, workplace observations and video analysis with design interventions" ([13], p. 332).

Design in this research is conceived "as being part of a larger and inevitable cycle of observing use, developing requirements (formal or informal), designing, building and again observing" [14]. In this framework, evaluation and use are seen as being an integral part of the design process and not terminal stages. In particular the evaluation, was organised by introducing a prototype in an on ongoing activity. It is not a set of metrics about the system that have been evaluated but the possible roles of novel technology in participants' activities. As Bannon [14] notes:

"a careful systematic account of what happens in particular settings when a prototype or system is installed, and how the system is viewed by the people on the ground, can provide useful information for 'evaluating' the system and its fitness for the purpose it was designed."

With this approach we organized a field trial in a real setting providing the environment for AR painting for 8 groups of architecture students (16 students). The teams of students used the environment to carry out one of their course assignments. While the course included creating models and collecting material over a whole semester the trail was organized over a period of two weeks. Over this period the teams of students took turns to use of the environment to produce a final presentation. Each team carried out three or four sessions of several hours in the environment and presented their work in a final plenary session at the end of the trial.

Each of the 8 student groups ( 2 students each) was asked to carry out an analysis of one of the 'icons' of modernist architecture from a selection of "Villas" in the city. They were required to read texts reflecting original and contemporary views on these buildings. They had to build two models 1:50 and 1:20 in scale (of an interesting spatial detail) and use our AR environment for analyzing scale and materiality. They worked with textures expressing the original ideas of the architects as well as with material of their own choice, exploring how materiality and context change the meaning of the building. Each student group was given a brief training of 4 minutes to learn how to operate the environment. Besides this brief training, the groups very rarely required support during their work sessions. A researcher observed the sessions 
making video recordings, which were used as material for interaction analysis. In addition, each group participated in semi-structured interviews where both members were interviewed simultaneously to collect the participants' views on the benefits and challenges of the environment.

\subsection{Working with Immersive and Mixed Media}

Each group worked with different physical models and different digital material. The use of the environment also varied significantly. While generally all the groups worked to produce different configurations of textures and background for a model, the following emerging and unanticipated uses were observed:

- installing a second Texture Brush to project textures from two directions, e.g. from the top and change the appearance of the floors or the roof while at the same time 'painting' the façade,

- using a "portable mouse"- a wireless mouse mounted on top of a cardboard box -as there was only one brush this was used especially for operating a second Texture Brush.

- taking pictures with a high resolution digital camera all around the model and also capturing the background,

- making and recording walkthroughs in the model using a web camera,

- using pictures or videos of the models on a large screen, playing with dimensions of small details of the models.

The students rarely used simple colors for painting, but applied colored textures, snapshots or even videos to their models. This notion goes beyond simply enriching a physical artifact by linking it with content in different media. In the case of the Texture Brush the link is such, that the properties of the object itself can be changed, by applying color, inserting movement, varying its dimension in relation to other objects in the physical space, varying context using multiple projections in the background. The participants were generally positive about the environment, expressing interest in working with it more in their studies. They also appreciated the immersiveness of the representations given by the possibility of using multiple large screens in the background. Another benefit was the possibility to rapidly create collages of textures and backgrounds for a model and the possibility to flexibly change it by loading and saving configurations.

\subsection{Spatial Distribution of Interface Components}

One of the distinctive properties of tangible interfaces is the importance of the spatial organization of users and computer equipment [15]. Through our system, users become immersed in the user interface and the architectural model, but limitations were imposed by our design solutions and the enabling technologies. In our system, the spatial configuration of the scanner, the brush, the model, the projectors, the mouse, and the poster can be re-organized by users so that they too, in a sense become part of the spatial organization. As foreseen in the TUI literature, the spatial distribution of a 
tangible interface carries many benefits arising from the exploitation of human visiospatial and motor capabilities. However, e observed many problems and corresponding workaround practices due to difficulties in spatial distribution. We here report and elaborate on four design challenges for others to learn.

First, the visibility of interface components is crucial to afford capabilities in different situations. A special challenge arises from the fact that the model, users, and tools can occlude each other in the tangible computing environment. We observed many times an interruption in work caused by searching for the correct tool. The visibility of tools can be accounted for by thinking about dedicated places in the environment for tools and preventing suboptimal places. If there are dedicated areas, such as the desktop in our example, and they can be left in no other place, then users will have a good idea on their probable locations all the time. However, this solution must be pitted against the goal of being able to tailor and configure tangible interface components according to the task at hand.

Second, referring to the objects of work is crucial in collaboration. We observed our users having difficulties in knowing to which part of the model some textures in a poster referred to, leading to problems in establishing a shared context or common ground regarding those objects (see [21]). In addition to difficulties in knowing the reference between the physical and the digital, users had difficulties in referring to tangible objects in talk-for example, referring to a particular projector, as they had no known or legible name. Therefore, we suggest considering giving legible names to tangible interface components-"Projector 1", "Scanner" etc. Moreover, we recommend explicating the reference from the physical to the digital where possible (e.g., stating with a picture to what part of the model a texture on the poster refers to), and being consistent in naming conventions across the physical and digital.

Third, multitasking in a tangible computing environment such as our system differs significantly from desktop interaction. We observed a user using the barcode scanner, the barcode leaflet, and the brush at the same time-which is obviously quite difficult with only two hands. To support multitasking, rapid switches between tools and tasks must be enabled, by aiming for all necessary tools to be within arms reach and readily usable without more interaction steps than picking up the tool. Our system, for example, had only very limited space for the mouse, which forced some users to pickup a piece of cardboard to extend the pad; likewise dedicated areas could be provided for different tools to be left close to the model. Therefore, to support fluent division of cognitive and motor capabilities, we suggest reflecting the possibility of designing for easily movable objects that can be held in different hands and nearby physical locations. For example, instead of having the palette projected on the desk, which forces the user to constantly shift attention and move between the model and the palette, it could be either integrated into the physical brush or into a separate tool similar to real painting palettes. When projecting the Texture Brush from above, for example, the palette would be projected onto the table or even onto the ground, requiring at times difficult postures from the participant in order to interact with the menu (Figure 3 right). Multimodality and rapid access solutions would also support ergonomic working postures and prevent injuries from long-term use. 

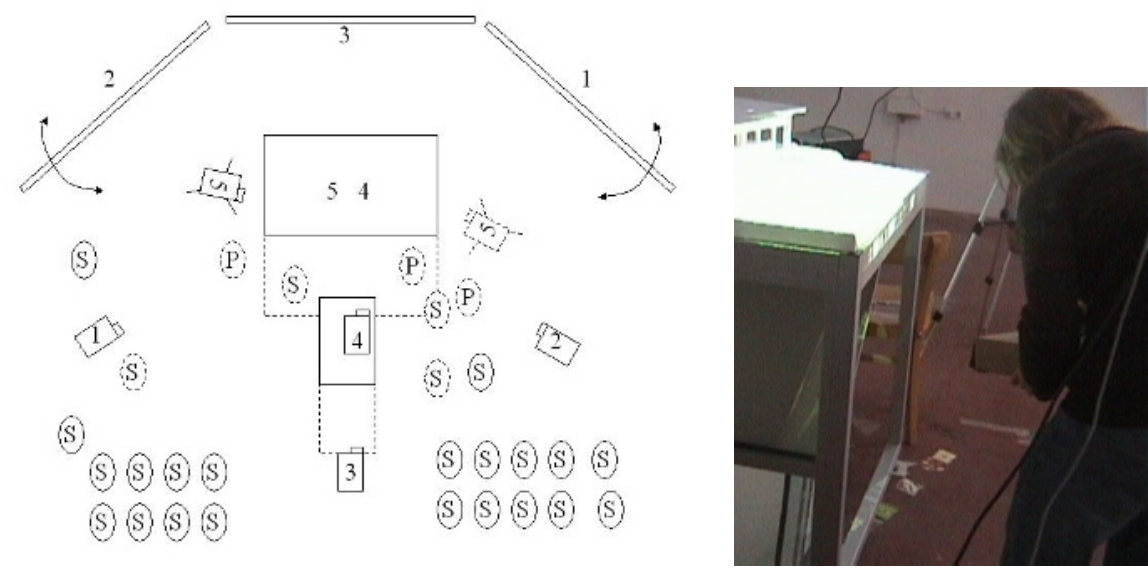

Fig. 3. Left: The physical arrangement of participants and components of the environment in a mixed media presentation. The letter "P" indicates participants operating the environment, the letter " $\mathrm{S}$ " indicates spectators. Numbers indicate 5 different projectors used for different purposes. Projectors 1,2,3 are used to provide a stage for the model. Right: a participant bending to operate the palette which is projected on the floor.

Finally, the spatial configuration of projectors is a challenge unique to tangible AR environments (Figure 3 left). They are essential for enabling the intermeshing of the digital and the physical, but they differ from other components in the fact that they are merely passive enablers, once set up and running, their initial configuration will most probably not be changed during one project session. Another bottleneck hindering initiation is how to restore the physical configuration. We made several observations that may inspire new ideas on how to improve the configurability and restoration of configurations. First, the placement of the projectors must enable convenient group work around the model, with minimum projected shadows due to a user standing in front of the beam. We observed some cases where one user had to command another to move away from the beam. Moreover, it is important that the projectors can reach parts of the model important for the work, different floors or balconies for example, and therefore their placement is significant. Second, the initial set up of the projector is mostly trial and error, and takes considerable time to learn. Our users used adhesive tape to mark the $\mathrm{x}$-y position of their projectors in the room and even to mark the height of the projector. Here, in addition to providing support for marking projector setups in the environment by using group-specific stickers etc, we suggest considering preparing ready-to-hand templates, maybe printed on paper or manuals, for wellknown projector configurations to help reduced the initial overload of projector configuration with common types of architectural models. Third, we noted some occasions where users wanted to turn the table, to work on the model from a different perspective, but this was not supported. Our projectors could not automatically adjust the beam to tilted or twisted surfaces, but such projectors now exist. 


\subsection{Synchronous Collaboration on and Around the Model}

In the architectural domain, it is imperative that collaborative practices and turntaking are supported by the system. We here report the lessons learned from the user study.

In all the sessions the environment was operated simultaneously by at least two participants. By "operated" we mean that at least two people were active most of the time carrying out tasks around the model. We observed a variety of collaborative work arrangements ranging from tight or loose collaboration to performing separate tasks on the same model, for example:

- One participant operating the barcode configuration and media posters and another participant painting with the physical brush. In these cases participants discuss which textures to make available on the palette or what kinds of backgrounds to configure in the background (tight collaboration).

- One participant painting the model from one direction (front) another participant painting the model from another direction (above). In these cases, participants engage in brief and sporadic discussions on parts of the models where their work meets up or on the general concept of the model.

- While the one participant changes painted textures and backgrounds the other documents the changes, creating different pictures from the same view.
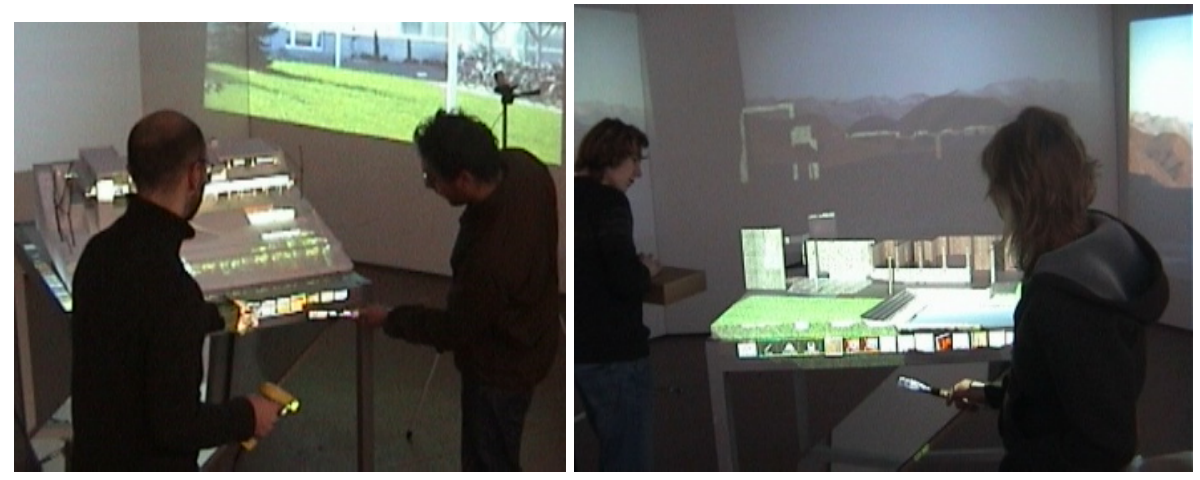

Fig. 4. Different types of collaborative arrangements

First, in our system, the texture that is worked on is shown as a cursor projected on the model. The cursor expresses the current target of operations. This pointer metaphor is adopted from desktop-based paint applications. In order to make it possible to perform operations with the scanner without holding the brush, the cursor is persistent, always there where it was last left. On one hand, this solution promotes shared understanding among group members on the current focus and status of the collaboration, as only one user can operate the system and others know what is happening. On the other hand, one-cursor solutions effectively prevent simultaneous work on the model. We observed that due to this shortcoming, others were rendered non-users of the system, although they were actively commenting on, instructing, or documenting 
the work. Currently, separate installations of the software have to be set up for separate projectors to enable working on different parts of the model. Here, in addition to the double set up of software being tedious, the system still did not enable simultaneous work on the same projection, which is what some groups wanted to have. Our suggestion is to consider multi-pointer solutions. These pointers should be distinctive and could incorporate indications of the brush size and shape and selected texture into the cursor icon. This could help in following what others are doing without extensive questioning and verbalizing. Moreover, authorship could be represented in the icon to communicate to others, who is responsible for that particular cursor.

\subsection{The Brush, Its Metaphor, and the Portable Mouse}

The brush as an interface tool in the setup was used for many purposes: painting, selecting options from the palette, drawing polygons by selecting, erasing paint, zooming and rotating a texture, and issuing commands with a press of a button. This interaction was based on hand movements and button presses, and extended the traditional uses of a brush and a palette remarkably (those of picking paint from a palette and then placing it onto a canvas). It is therefore worthwhile considering how well the brush and palette metaphors align with these uses and what are the implications of bending the concept of a traditional brush.

Two different types of questions emerge from this consideration. The first one is about how intuitive is the concept of a brush providing also non-obvious features. Some of these functions are natural, such as painting with the brush, but zooming is a feature that has to be taught since it is not a part of the brush metaphor. Our data indicates, however, that users are able to be quite adaptive in extending the metaphor. For instance, the technical limitation of not being able to touch surfaces with the brush was exploited opportunistically by painting from a distance of one meter. Another user visualized a Las Vegas like hotel with neon signs, by using textures as separate images. He placed scanned logos onto a surface and erased the paint around the logos, creating a sticker-like visual effect. The use of the palette in carrying out all the menu-related tasks was also natural to the users, although traditional palettes do not contain menus. Therefore, it seems that strict accordance with metaphors is not always required to produce intuitively usable interaction tools. The brush and palette metaphors allowed and constrained the modes of interaction into which all the necessary functionalities could be embedded. For instance, the thinness of a brush did not allow for including a menu into it, but enabled the users to paint areas that would have been unreachable with other tools. The palette metaphor, as a container of textures can be naturally extended to provide other types of selectable functionalities as well.

A portable wireless mouse on a cardboard box was used in some cases (two participants) as a substitute for the physical brush. It was used mainly to manipulate the second Texture Brush from above. Therefore, the palette for the mouse was projected onto the floor or onto the table. The primary difference in using the mouse instead of the brush was that paint was applied to the model from a distance and based on where the user saw the cursor, not by reaching towards it to position where the paint was to be applied. However, positioning the mouse very close to the model is important in order to support visibility and hand-eye co-ordination. Probably, the most important phenomenon was that mouse movements on the box were not easily mapped to cursor 
movements on the model. While when we operate the mouse on a desktop computer our orientation to the screen does not change, the participants in our case moved around the model, adopting different positions and orientations frequently resulting in a "misalignment" of the mouse with the cursor.

\section{Discussion and Conclusions}

The main motivation of tangible user interfaces (TUIs) is to move meaningful interaction from digital to physical. Physicality can characterise human-computer interaction in many different and unprecedented ways, including spatiality, artefacts, and various other manifestations of computational resources [16]. While a variety of tangible interfaces and applications are merely presented as demos at conferences and only operated by researchers, there is a growing need for field evaluation in realistic settings. In this paper, we presented an environment for augmented reality painting of physical models and reported on its evaluation in a field trial in a setting with several architecture students working on course projects.

We observed several positive properties of TUIs in the field study. However, our work also revealed many challenges, which remain for future work on making TUIs more usable and attractive. The most vital is the challenge of the distributed interface-the physical, spatial, and computational separation of interface components. This distribution cannot be arbitrary but must possess the capability to act meaningfully through the combination of individual multiple input/output devices so that the outcome also makes sense at the level of the system [18]. In a TUI environment like ours, this implies cognitive and creative processes being distributed and coordinated across the members of the group, these processes also being distributed over time and space and at least partly mediated by computer and physical artefacts [17]. A central corollary challenge is to turn the public availability of embodiment (cf. [19]) into real collaborative features of the interface. In our system, collaboration around the model was limited by the single-cursor, single-tool nature of the system. In addition to considering multi-cursor multi-tool designs, we saw that the visibility, labeling, affordance, and accountability of interface components is necessary. At the individual level, multimodality and efficient use of cognitive resources is necessary, also for ergonomical reasons. Finally, intertwining the digital and physical in a bi-directional manner poses a problem for future research. In our field study interfacing the two worked mostly mono-directionally. For example, if the user changed the position of the model, the projection did not adjust accordingly, as the projectors were not sensitive to changes in the real world. Bi-directionality would promise easier set-ups and in-session configurability of the system, but would require rather complex on-line video signal processing to work.

Similar systems have been developed that concentrate on one aspect of the interaction, offering more sophisticated features, such as the possibility of moving, real time, the physical objects and the projected textures using tracking technology $[3,4]$. Other studies that compare in detail 2D and tangible interfaces [9, 10] merely state which one performs better using some general criteria (e.g., memory in spatial layout tasks [9] or trial time and user operations [10]). Other work that reports on observational studies of current practices to inform the development of tangible interfaces, provides 
the motivations but only vague indications of the features to be implemented [12]. Finally heuristic evaluations have been proposed for ambient displays which are weakly applicable in the case of tangible interfaces.

Most relevant to our research are the system guidelines for co-located collaborative work on a table-top display [20]. Our study contributes specific knowledge on how to extend the guidelines for tangible interfaces. In particular, our study contributes to the guideline support fluid transition between activities, for tangible interfaces, proving the trade-off between the advantages of specializing tangible interaction (the barcode scanner for "phycons", the physical brush for painting, etc.) and the disadvantages, for fluid transitions, of distributing interfaces across different platforms and tools. For the guidelines support interpersonal interaction, transitions between personal and group work and simultaneous user actions our study highlighted another trade-off between supporting clear divisions of labor and supporting synchronous collaboration while accessing simultaneously shared resources. For example TUIs in our case supported a clear division of labor (one participants selecting textures and backgrounds and the other one applying and modifying textures with the brush, or two participants painting simultaneously), however, with limitations due to missing groupware features and single-tool approaches (a single barcode scanner and a single brush were available). For other guidelines such as support the use of physical objects, shared access to physical and digital objects the study demonstrates not only the need to integrate physical objects and affordances (physical models, sheets of papers with icons and visual codes) but the opportunity of mixing digital and physical objects (the model painted with digital textures). Finally, our study provides additional candidate guidelines (requirements) for tangible computing environments. It was possible through a field study to show evidence of the need to support, not only a single interaction scenario, but a whole activity cycle of which painting digital textures might be just a phase. The field study helped us to gain a more "ecological or systemic" perspective, showing the need to support the upload, retrieval and saving of mixed media configurations and also the opportunity to create immersive environments that extended out of a "table-top" with multiple and large scale projections.

\section{References}

1. Abowd, G. D., Mynatt, E. D., Charting Past, Present, and Future Research in Ubiquitous Computing, ACM TOCHI, Vol. 7, No. 1, March (2000) 29-58

2. Abowd G D., Mynatt E D., Rodden, T., The Human Experience. In: IEEE Pervasive Computing archive Volume 1, Issue 1 (January 2002) 48 - 57 .

3. Raskar, R., Welch, G., Chen, W.-C.: Table-Top Spatially-Augmented Reality: Bringing Physical Models to Life with Projected Imagery. Proc. IWAR'99. IEEE (1999) 64-73

4. Bandyopadhyay, D., Raskar, R., Fuchs, H.: Dynamic Shader Lamps: Painting on Movable Objects. Proc. ISAR'01. IEEE Computer Society, New York (2001) 207-216

5. Schmudlach, K., Hornecker, E., Ernst, H., Bruns, F.W.: Bridging Reality and Virtuality in Vocational Training. CHI'00 Interactive Posters. ACM Press, New York (2000) 137-138

6. Underkoffler, J., Ishii, H.: Illuminating Light: An Optical Design Tool with a LuminousTangible Interface. Proc. CHI'98. ACM Press, New York (1998) 542-549 
7. Ryokai, K., Marti, S., Ishii, H.: I/O Brush: Drawing with Everyday Objects as Ink. Proc. CHI'04. ACM Press, New York (2004) 303-310 Mankoff, J., Dey, A.K., Hsieh, G., Kientz, J., Lederer, S., Ames, M.: Heuristic Evaluation of Ambient Displays. Proc. CHI'03, ACM Press, New York (2003) 169-176

8. Mankoff, J., Dey, A.K., Hsieh, G., Kientz, J., Ames, M., Lederer, S.: Heuristic evaluation of ambient displays. CHI 2003, ACM Press, CHI Letters 5(1): 169-176

9. Huang, C.-J.: Not Just Intuitive: Examining the Basic Manipulation of Tangible User Interfaces. CHI'04 Late Breaking Results. ACM Press, New York (2004) 1387-1390

10. Fjeld, M., Guttormsen Schär, S., Signorello, D., Krueger, H.: Alternative Tools for Tangible Interaction: A Usability Evaluation. Proc. ISMAR'02. IEEE (2002) 157-166

11. Fitzmaurice, G.W., Buxton, W.: An Empirical Evaluation of Graspable User Inter-faces: Towards Specialized, Space-Multiplexed Input. Proc. CHI'97. ACM Press, 1997, 43-50.

12. Brereton, M., McGarry, B.: An Observational Study of How Objects Support Engineering Design Thinking and Communication: Implications for the Design of Tangible Media. Proc. CHI'00. ACM Press, New York (2000) 217-224

13. Trigg RH, Blomberg J \& Suchman L (1999). Moving document collections online: The evolution of a shared repository. Proceedings of the Sixth European Conference on Computer Supported Cooperative Work (ECSCW'99). S. Bødker, M. Kyng, K. Schmidt (eds.). Dordrecht, The Net-herlands: Kluwer, 331-350.

14. Bannon, L. J.: Use, Design and Evaluation - Steps towards an Integration. In: Shapiro, D., Tauber, M., Traunmueller, R. (eds): The Design of Computer-Supported Cooperative Work and Group-ware Systems. Amsterdam: North - Holland, (1996) 423-442.

15. Dourish, P.: Where the action is: the foundations of embodied interaction. MIT Press 2001

16. Jacucci, G.: Interaction as Performance. Cases of Physical Interfaces and mixed media design. Doctoral Thesis, University of Oulu, Acta Universitatis Ouluensis. (2004)

17. Hollan, J., Hutchins, E., and Kirsh, D.: Distributed cognition: toward a new foundation for human-computer interaction research ACM (TOCHI), (2000) pp.174-196.

18. Binder, T., De Michelis, G., Gervautz, M., Jacucci, G., Matkovic, K., Psik, T., Wagner, I.: Supporting Configurability in a Mixed Media Environment for Design Students. In: Special Issue on Tangible Interfaces in Perspective, Pers and Ubiq Comp, Journal, Volume 8 , Issue 5, Springer Verlag (September 2004) 310 - 325.

19. Robertson, T.,: The Public Availability of Actions and Artefacts', Computer-Supported Cooperative Work, vol. 11, (2002)

20. Scott, S., Grant, K., Mandryk, R.,: System Guidelines for Co-Located, Collaborative Work on a Tabletop Display. In: Proceedings of ECSCW'03, European Conference of Computer Supported Cooperative Work 2003, Helsinki Finalnd, September 14-18, Kluwer, 2003.

21. Kraut, R. E., Fussell, S. R., Siegel, J.: Visual information as a conversational resource in collaborative physical tasks. Human-Computer Interaction, 18, 13-49, (2003) 